\title{
35-GHz Wideband Vertical Off-Center-Fed Dipole and Array in LTCC Technology
}

\author{
Ming $D U^{1}$, Jun $X U^{1}$, Xiao DING ${ }^{1}$, Jiangping $C A O^{2}$, Jianhua $D E N G^{2}$, Yuliang DONG ${ }^{2}$ \\ ${ }^{1}$ School of Physical Electronics, University of Electronic Science and Technology of China, Chengdu, China \\ ${ }^{2}$ National Institute of Measurement and Testing Technology of China, Chengdu, China
}

mingdu_uestc@163.com,xujun@uestc.edu.cn,xding@uestc.edu.cn,jh_deng@126.com,1878281644@qq.com, dongyull@163.com

Submitted June 8, 2017 / Accepted October 18, 2017

\begin{abstract}
A 35-GHz wideband low-temperature co-fired ceramic (LTCC) vertical off-center-fed dipole antenna is presented. The widened bandwidth of the dipole antenna is achieved by paralleling a ring-shaped stub placed above the ground with a vertical off-center-fed dipole. The fabricated dipole antenna with a dimension of $7.5 \times 19 \times 0.94 \mathrm{~mm}^{3}$ exhibits a measured $-10-\mathrm{dB}\left|S_{11}\right|$ bandwidth of $25.4 \%$ covering the frequency range of 31.2 to $40 \mathrm{GHz}$ and a measured maximum gain of $4.4 \mathrm{dBi}$ at $32 \mathrm{GHz}$ with a fluctuation of $1.7 \mathrm{~dB}$ over 31 to $39 \mathrm{GHz}$ frequency range. Then, a $2 \times 2$ array was designed. The array with a dimension of $15 \times 25 \times 0.94 \mathrm{~mm}^{3}$ exhibits a measured $-10-d B\left|S_{11}\right|$ bandwidth of $28.6 \%$ covering the frequency range of 30 to $40 \mathrm{GHz}$ and a maximum gain of $10.37 \mathrm{dBi}$ at $31 \mathrm{GHz}$ with a fluctuation of $1.8 \mathrm{~dB}$ over 31 to $39 \mathrm{GHz}$ frequency range. The radiation performances of the dipole antenna and its array were also measured. Good agreement is obtained between the simulations and measurements.
\end{abstract}

\section{Keywords}

Dipole antenna, wideband; antenna array, millimeterwave $(\mathrm{mmW})$, low-temperature co-fired ceramic (LTCC)

\section{Introduction}

Millimeter-wave $(\mathrm{mmW})$ antennas and arrays with wideband and high integration are receiving increasing demand recently. The low-temperature co-fired ceramic (LTCC) technology is a good option for integration at $\mathrm{mmW}$ because of light weight, low profile, compactness and excellent high frequency performance. Over past few years, antennas for wideband and high integration based on LTCC have been investigated such as patch antennas [1-6], dipole antennas [7], [8], helical antennas [9], [10], horn antennas [11], etc. Among them, the dipole antenna has the simplest structure and implementation.

Simply speaking, the dipole antenna can be classified into two types: horizontal-fed dipole antennas [12-16] and vertical-fed dipole antennas [17], [18]. For easy understanding, Figure 1 shows the structures of the horizontal-fed and vertical-fed dipole antennas. It can be seen that the horizontal-fed dipole antenna is similar with the edge-fed patch antenna. The dipole and the feeding structure are easy to be printed in the same substrate. The vertical-fed dipole antenna is similar with the probe-fed patch antenna. The dipole and the feeding structure are hard to be printed in the same substrate.

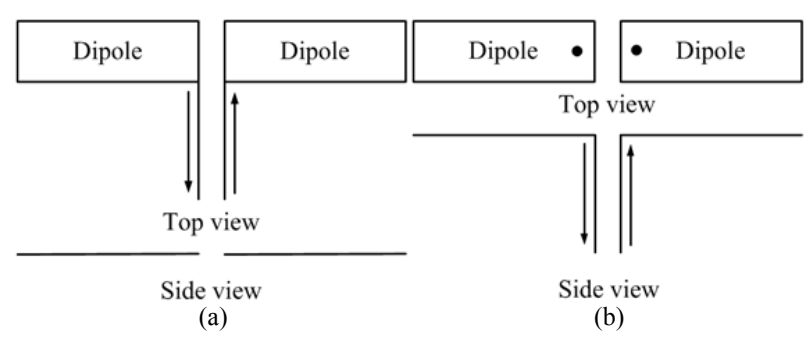

Fig. 1. Horizontal-fed and vertical-fed dipole antennas: (a) horizontal-fed dipole antenna, (b) vertical-fed dipole antenna.

Compared with horizontal-fed dipole antennas, vertical-fed dipole antennas have less coupling between the radiator and the feeding structure for the orthogonal relation between them. Some vertical-fed dipole antennas with wideband were also presented [17], [18]. It should be noted that a vertical wideband balun is always needed to feed the dipole in them. Therefore, it can be said that all these dipole antennas are not suitable for integration using LTCC, because only blind vias can be implemented in vertical direction which causes hard realization of the vertical wideband balun. It is not so easy to achieve an integrated wideband vertical-fed dipole antenna based on LTCC.

A 60-GHz wideband LTCC vertical stripline-fed dipole array with a $-10-\mathrm{dB}\left|S_{11}\right|$ bandwidth of $15.7 \%$ was reported in [7]. For widening the bandwidth, two resonances were generated by the dipole and feeding aperture respectively. Nevertheless, this technology is not suited for a microstrip-fed dipole which benefits from easy integration of active components, since the resonance generated by the feeding aperture will produce an omnidirectional radiation. 
In [8], we present a wideband vertical dipole antenna fed by substrate integrated waveguide (SIW) using LTCC technology working at Ka band. A transverse slot is etched on the upper ground of the SIW for widening the bandwidth of the dipole antenna. Therefore, the slot is in series with the dipole, which can change the input impedance. However, the SIW feeding structure merits low loss but incompactness because of its large size. The slot is placed on the feeding structure, which is also a reason of the incompactness of the feeding structure. Besides, the bandwidth is widened a little, because the slot just can change the input impedance a little.

In this paper, a 35-GHz LTCC vertical off-center-fed dipole antenna fed by microstrip is proposed. For achieving wideband, a ring-shaped stub was paralleled with a vertical off-center-fed dipole. The feeding aperture is not designed as a radiator. Therefore, the radius is designed as small as possible to reduce the radiation back lobe. The ring-shaped stub is not placed in the feeding structure which is microstrip. Therefore, the feeding structure is compact. Besides, the ring-shaped stub resonates near the working band, therefore the input impedance can be changed greatly. As a result, the bandwidth can be widened more effectively. Then, a $2 \times 2$ planar array is formed for high gain. The proposed array exhibits wideband and high-gain characteristics.

\section{Antenna Element}

\subsection{Antenna Element Configuration and Performances}

Figure 2 shows the configuration of the proposed dipole antenna. The multi-layer LTCC substrate used is Ferro A6-M with dielectric permittivity $\varepsilon_{\mathrm{r}}=5.9 \pm 0.2$ and loss tangent $\tan \delta=0.002$ at $35 \mathrm{GHz}$. Each LTCC layer has a thickness of $0.094 \mathrm{~mm}$. The antenna is composed of four parts: one dipole with two arms of different lengths, two parallel signal vias, a ring-shaped stub and feeding microstrip. The arm 1 is connected to the microstrip through a circular aperture on the ground by the signal via 1, while the arm 2 is connected to the ground by the signal via 2 . The length of the two signal vias is about $\lambda_{\mathrm{g}} / 4\left(\lambda_{\mathrm{g}}\right.$ is the wavelength in the LTCC substrate at $35 \mathrm{GHz}$ ). The ringshaped stub placed above the ground consists of two half rings lying on two different layers with end connection implemented by a blind via. The characteristic impedance of the microstrip is $50 \Omega$. The detailed dimensions of the proposed dipole antenna are shown in Tab. 1.

The two signal vias can be seen as a parallel double transmission line whose characteristic impedance can be expressed as (1) [19]:

$$
Z_{0}=\frac{\eta_{0}}{\pi} \ln \left[\frac{D}{d}+\sqrt{\left(\frac{D}{d}\right)^{2}-1}\right]
$$

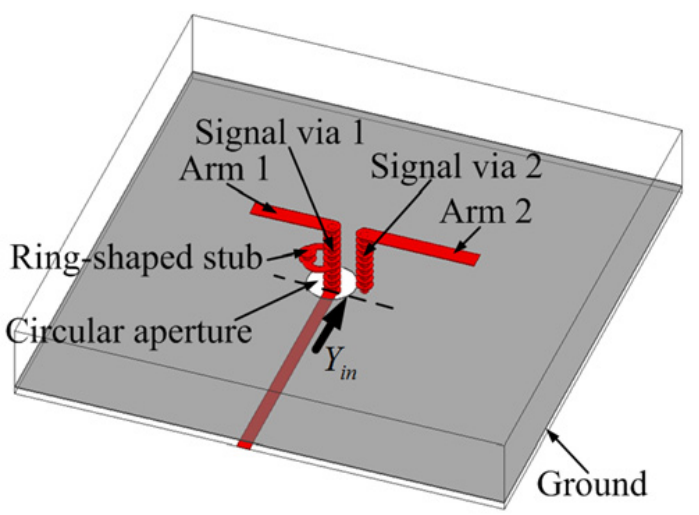

(a)
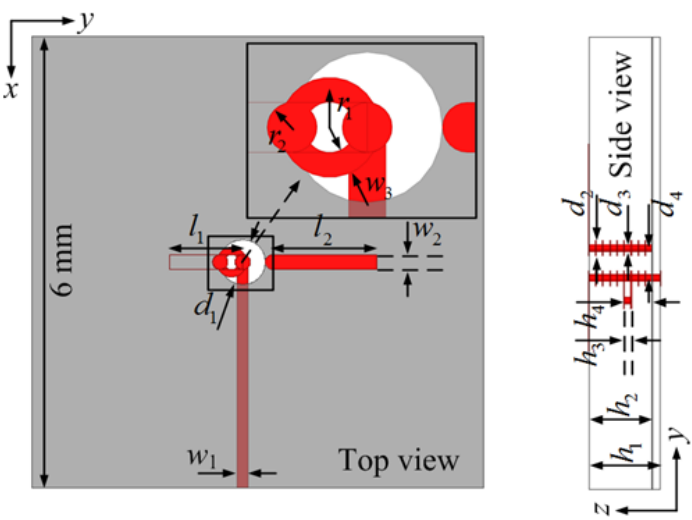

(b)

Fig. 2. Configuration of the proposed dipole antenna: (a) 3D view and (b) top and side views.

\begin{tabular}{|c|c|}
\hline Parameter & Value (mm) \\
\hline$l_{1}$ & 0.97 \\
\hline$l_{2}$ & 1.38 \\
\hline$w_{1}$ & 0.15 \\
\hline$w_{2}$ & 0.2 \\
\hline$w_{3}$ & 0.1 \\
\hline$d_{1}$ & 0.6 \\
\hline$d_{2}$ & 0.2 \\
\hline$d_{3}$ & 0.1 \\
\hline$d_{4}$ & 0.4 \\
\hline$h_{1}$ & 0.94 \\
\hline$h_{2}$ & 0.846 \\
\hline$h_{3}$ & 0.094 \\
\hline$h_{4}$ & 0.282 \\
\hline$r_{1}$ & 0.14 \\
\hline$r_{2}$ & 0.1 \\
\hline
\end{tabular}

Tab. 1. Detailed dimensions of the proposed dipole antenna.

where $\eta_{0}$ is the wave impedance, $D$ is the spacing between the two lines and $d$ is the diameter of each line. The radius of the circular aperture should be as small as possible because if it is large, the circular aperture can radiate effectively which will cause a large radiation back lobe. There is a gap between the circular aperture and the signal via 1 . 
The minimum width of a gap should be wider than $0.15 \mathrm{~mm}$ because of the limitation of the fabrication. For easy fabrication, $0.2-\mathrm{mm}$ gap was selected. As a result, the radius of the circular aperture is $0.3 \mathrm{~mm}$. In addition, the distance between the two signal vias should be as short as possible, because one signal via is connected to the feeding microstrip and the other one is connected to the ground. If the distance is long, the amplitude of the currents on the two signal vias will be different which will cause an unbalanced feeding of the dipole. Therefore, this distance $D$ was chosen to be $0.4 \mathrm{~mm}$. According to formula (1) and the other dimensions shown in Tab. 1, the characteristic impedance of the two signal vias should be about:

$$
Z_{0}=\frac{120 \pi}{\pi \sqrt{5.9}} \ln \left[\frac{0.4}{0.1}+\sqrt{\left(\frac{0.4}{0.1}\right)^{2}-1}\right] \approx 100 \Omega .
$$

As a result, the input impedance of the dipole should be about $200 \Omega$ to get impedance matched according to the quarter-wavelength impedance transformation theory (the characteristic impedance of the feeding microstrip is $50 \Omega$ and the length of the signal vias is about $\lambda_{\mathrm{g}} / 4$ ). As mentioned in [20], the input impedance of a center-fed halfwavelength dipole is about $73 \Omega$ and the off-center feeding technology can increase the input impedance rapidly. Therefore, the proposed dipole is off-center-fed.

For illustrating the effect of the stub, the simulated $\left|S_{11}\right|$ s of the antennas with and without the stub are plotted in Fig. 3(a). As observed, the antenna without the stub has only one resonant pole at $32.5 \mathrm{GHz}$ and the $-10-\mathrm{dB}$ $\left|S_{11}\right|$ bandwidth is narrower than $15 \%$ covering the frequency range of 30.6 to $35.8 \mathrm{GHz}$. On the other hand, the antenna with the stub has two resonant poles at 31.8 and $37.7 \mathrm{GHz}$. The $-10-\mathrm{dB}\left|S_{11}\right|$ bandwidth is $27.9 \%$, covering the frequency range of 30.3 to $40 \mathrm{GHz}$, which is almost twice as wide as that of the antenna without the stub.

\subsection{Working Principle}

For understanding how the bandwidth of the antenna without the stub is widened, the comparisons of the simulated input admittances of the antennas with $\left(Y_{\text {in } \mathrm{t}}\right)$ and without the stub $\left(Y_{\text {in_d }}\right)$ are shown in Fig. 3(b). The position and direction of both input admittances are depicted in Fig. 2(a). As observed in Fig. 3(b), the $Y_{\text {in d }}$ is nearly $(0.02+\mathrm{j} 0) \mathrm{S}$ at $32.5 \mathrm{GHz}$, which corresponds to input impedance of $(50+\mathrm{j} 0) \Omega$. However, the re $\left(Y_{\text {in_d }}\right)$ decreases and the $\operatorname{im}\left(Y_{\text {in_d }}\right)$ becomes negative at higher frequencies, which deteriorates the impedance matching. Therefore, the bandwidth is narrow.

On the other hand, the antenna with the stub has better impedance matching at higher frequencies, while keeps good impedance matching almost unchanged at about $32.5 \mathrm{GHz}$. This is because the stub coupled with the circular aperture on the ground resonates as a parallel RLC circuit at about $32.5 \mathrm{GHz}$, while it resonates as a series RLC circuit at $43.9 \mathrm{GHz}$.

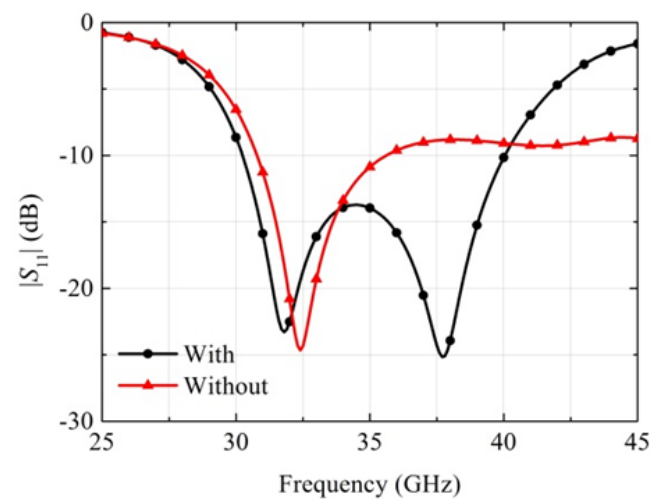

(a)

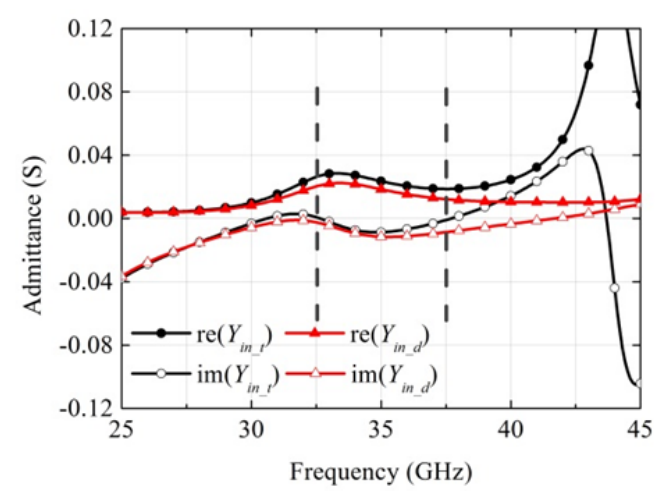

(b)

Fig. 3. Simulated (a) $\left|S_{11}\right| \mathrm{s}$ and (b) $Y_{\text {in }}$ s of the antennas with and without the stub.

For easy understanding, the equivalent circuits of the stub at 32.5 and $43.9 \mathrm{GHz}$ are proposed in Fig. 4(a). As shown, when the stub resonates as a parallel RLC circuit, the input admittance of the stub ( $\left.Y_{\text {in } \mathrm{s}}\right)$ is almost zero and $Y_{\text {in } \mathrm{t}}=Y_{\text {in d }}+Y_{\text {in s }} \approx Y_{\text {in d }}$, so the stub has little effect on the dipole. And when it resonates as a series RLC circuit, both the $\operatorname{re}\left(Y_{\text {in_s }}\right)$ and $\operatorname{im}\left(Y_{\text {in_s }}\right)$ become large. Besides, the $\operatorname{im}\left(Y_{\text {in_s }}\right)$ and the $\operatorname{im}\left(Y_{\text {in_d }}\right)$ are opposite in sign. Thus, the $Y_{\text {in } \mathrm{t}}$ is improved at higher frequencies. At $37.7 \mathrm{GHz}$, the $Y_{\text {in }}{ }_{-}$is increased to about $(0.02+\mathrm{j} 0) \mathrm{S}$. As a result, another resonant pole at $37.7 \mathrm{GHz}$ is generated while the resonant pole at $32.5 \mathrm{GHz}$ does not disappear and the bandwidth is widened.

A broadband equivalent circuit is also proposed as shown in Fig. 4(b). This equivalent circuit can work at all working band. The equivalent circuit is extracted by using the proposed working principle. The $R_{1}, L_{1}$ and $C_{1}$ are used for introducing the series RLC resonance at $43.9 \mathrm{GHz}$, the $R_{1}$, which is small, indicates the loss of the series RLC circuit. The $L_{2}$ is used for introducing the parallel RLC resonance at $32.5 \mathrm{GHz}$, and the $R_{2}$, which is large, indicates the loss of the parallel RLC circuit. The detailed values are: $R_{1}=10 \Omega, L_{1}=0.79 \mathrm{nH}, C_{1}=0.0165 \mathrm{pF}, R_{2}=200 \Omega$ and $L_{2}=0.69 \mathrm{nH}$.

The steps of the extraction of the equivalent circuit are:

1. Design the series RLC circuit and extract the values of the series RLC circuit. Make sure the series RLC 


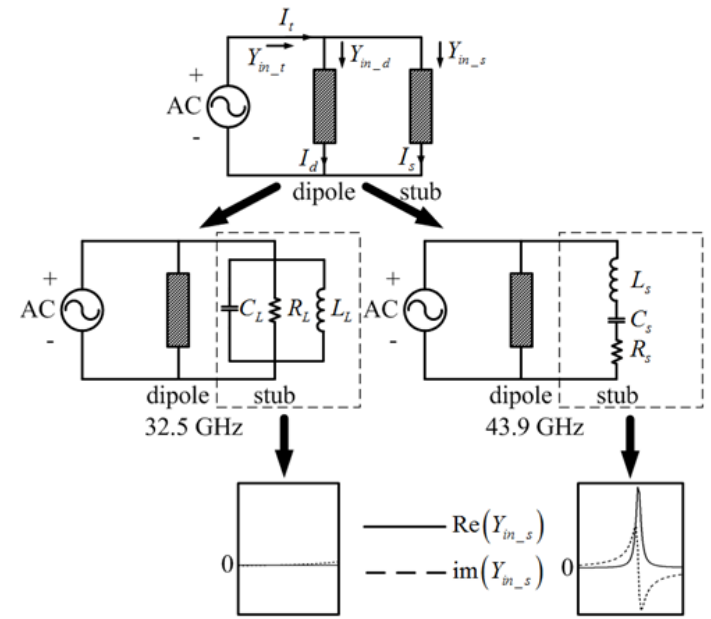

(a)

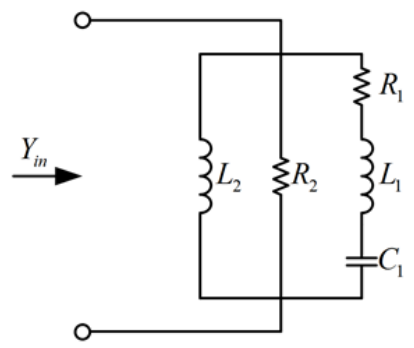

(b)

Fig. 4. Equivalent circuits of the stub: (a) working at 32.5 and $43.9 \mathrm{GHz}$, respectively and (b) working at all the working band.

circuit resonates at $43.9 \mathrm{GHz}$. The simulated $Y_{\text {in }_{-} \mathrm{s}}$ in this step is shown in Fig. 5(a). As observed, a series RLC resonance at $43.9 \mathrm{GHz}$ is generated.

2. Design the parallel RLC circuit and extract the values of $R_{2}$ and $L_{2}$. Make sure the parallel RLC resonance at $32.5 \mathrm{GHz}$ is generated. As observed in Fig. 5(a), the $\operatorname{im}\left(Y_{\text {in }}\right)$ is positive. So, an inductance $L_{2}$ is paralleled with the series RLC circuit to achieve the parallel RLC resonance at $32.5 \mathrm{GHz}$. The simulated $Y_{\text {in_s }}$ in this step is shown in Fig. 5(b). As observed, a parallel RLC resonance at $32.5 \mathrm{GHz}$ is generated while the series RLC resonance at $43.9 \mathrm{GHz}$ do not change mostly. This is because the impedance of the parallel RLC circuit at $43.9 \mathrm{GHz}$ is small, which can short the $R_{2}$ and $L_{2}$.

The simulated $\left|S_{11}\right|$ s and $Y_{\text {in }}$ of the antenna without the ring-shaped stub but combined with this equivalent circuit compared with the dipole antenna with the stub are given in Figs. 5(c) and (d). As observed, they agree with each other well, which proves the proposed working principle is reasonable.

To further verify the working principle, the current distributions on the circular aperture and two arms at different times are depicted in Fig. 6. $T$ is the period of oscillation at each frequency. The currents on the aperture at any time should be the sum of the travelling-wave currents introduced by the dipole for transferring energy and the standing-wave currents introduced by the stub for wide-

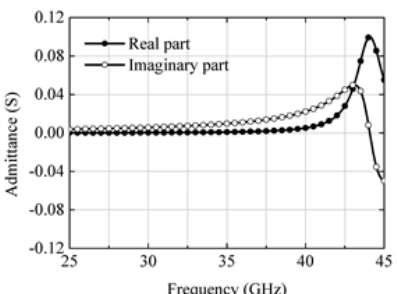

(a)

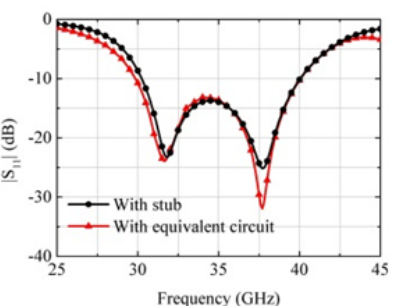

(c)

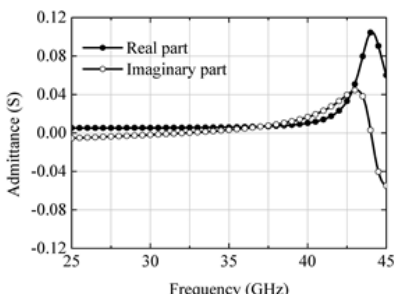

(b)

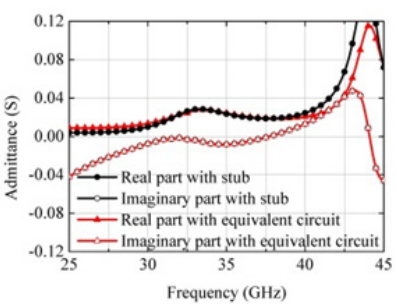

(d)
Fig. 5. Simulated results of the stub: (a) $Y_{\text {in } \mathrm{s}}$ in the first step, (b) $Y_{\text {in_s }}$ in the second step, (c) comparison of $\left|S_{11}\right| \mathrm{s}$ between the dipole paralleled with the stub and the total equivalent circuit and (d) comparison of $Y_{\text {ins }}$ between the dipole paralleled with the stub and the total equivalent circuit.

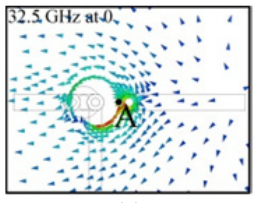

(a)

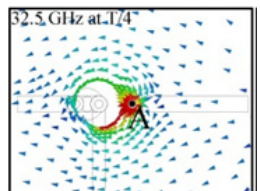

(d)

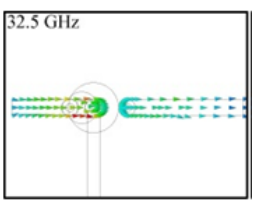

(g)

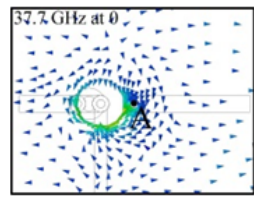

(b)

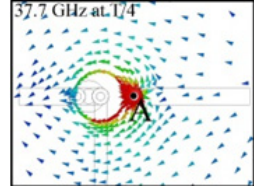

(e)

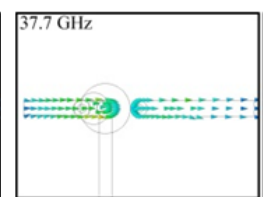

(h)

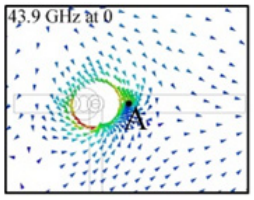

(c)

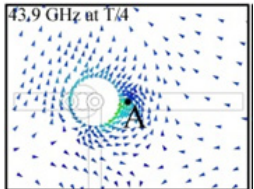

(f)

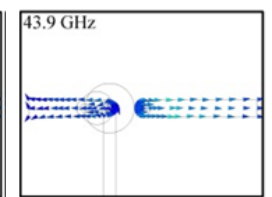

(i)

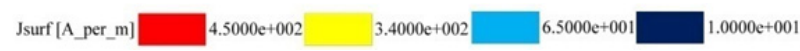

(j)

Fig. 6. Current distributions on the circular aperture and two arms: (a) $32.5 \mathrm{GHz}$ when $t=0$ on the aperture, (b) $37.7 \mathrm{GHz}$ when $t=0$ on the aperture, (c) $43.9 \mathrm{GHz}$ when $t=0$ on the aperture, (d) $32.5 \mathrm{GHz}$ when $t=T / 4$ on the aperture, (e) $37.7 \mathrm{GHz}$ when $\mathrm{t}=\mathrm{T} / 4$ on the aperture, (f) $43.9 \mathrm{GHz}$ when $t=T / 4$ on the aperture, (g) $32.5 \mathrm{GHz}$ on the two arms, (h) $37.7 \mathrm{GHz}$ on the two arms, (i) $43.9 \mathrm{GHz}$ on the two arms and (j) legend.

band (this is the same as $I_{\mathrm{t}}=I_{\mathrm{d}}+I_{\mathrm{s}}$, as shown in Fig. 4(a)). The travelling-wave currents must converge at point $A$ while the standing-wave currents may not. Moreover, when a parallel RLC circuit resonates, the currents on it should be small because of the large input impedance. On the contrary, when a series RLC circuit resonates, the currents on it should be large for the small input impedance. 
The currents on the aperture at the first resonance at 32.5 $\mathrm{GHz}$ converge at point $\mathrm{A}$ at both times, which means the travelling-wave currents are strong while the standingwave currents are weak. This also indicates that the stub resonates as a parallel RLC circuit at the frequency and the stub has little influence on the dipole. At the second resonance at $37.7 \mathrm{GHz}$, the currents on the aperture converge at point A when $t=T / 4$ and they do not converge at point A when $t=0$. The standing-wave currents and the travellingwave currents appear alternately, which indicates the frequency is near the series resonance pole and the stub has a great influence on the dipole. So the bandwidth is improved at higher frequencies while remaining unchanged at lower frequencies. The currents on the two arms are in the same direction and strong at 32.5 and $37.7 \mathrm{GHz}$, which indicates the two arms are the main radiator at both resonances. The currents at $43.9 \mathrm{GHz}$ do not converge at point $A$ at any times. And the standing-wave currents are stronger than that at $37.7 \mathrm{GHz}$. This is because the stub resonates as a series RLC circuit. at $43.9 \mathrm{GHz}$. As a result, the input impedance of the stub is small at $43.9 \mathrm{GHz}$, while the input impedance of the dipole is large. As a result, the dipole is shorted by the stub, which can be validated by the weak currents on the two arms as shown in Fig. 6(i). The currents on the two arms are also opposite.

\subsection{Parameter Study}

As shown in Fig. 7(a), the lengths of the two arms $l_{1}$ and $l_{2}$ have significant influence on the first resonance frequency. As $l_{1}$ is increased and $l_{2}$ is decreased while keeping the total length of the two arms unchanged, the first resonance frequency shifts to higher frequency while the second resonance frequency stays the same mostly. The effect of the width of the two arms on $\left|S_{11}\right|$ is illustrated in Fig. 7(b). Figures 8 and 9(a) plot the effects of the height over the ground $h_{4}$ and dimensions of the ring-shaped stub

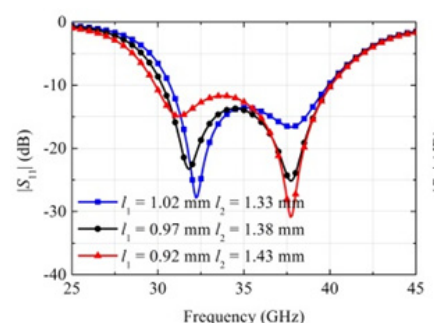

(a)

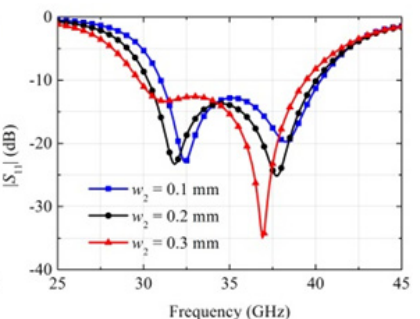

(b)
Fig. 7. Effects of $l_{1}, l_{2}$ and $w_{2}$ on $\left|S_{11}\right|:$ (a) $l_{1}$ and $l_{2}$; (b) $w_{2}$.

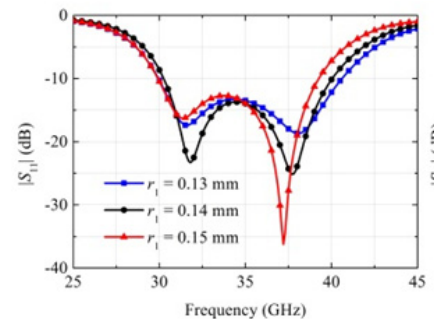

(a)

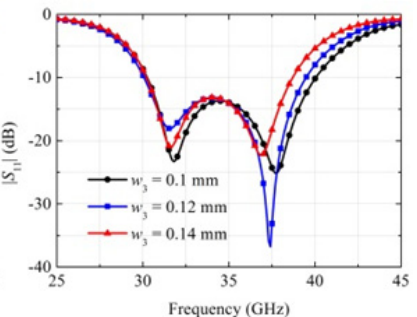

(b)
Fig. 8. Effects of $r_{1}$ and $w_{3}$ on $\left|\mathrm{S}_{11}\right|$ : (a) $r_{1}$ and (b) $w_{3}$.

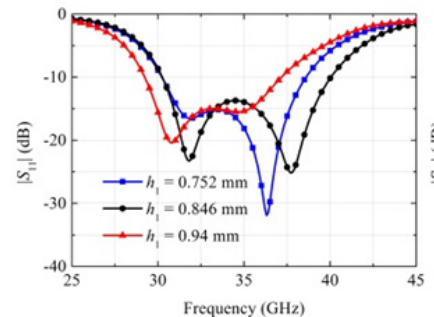

(a)

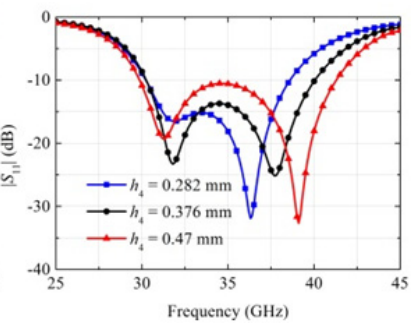

(b)
Fig. 9. Effects of $h_{1}$ and $h_{4}$ on $\left|S_{11}\right|$ : (a) $h_{1}$ and (b) $h_{4}$.

on $\left|S_{11}\right|$. With the increasing of the radius and width of the stub, the second resonance frequency converts to lower frequency while the first resonance frequency remains unchanged mostly. At the same time, when $h_{4}$ is increased, the second resonance frequency is transformed to higher frequency while the second resonance frequency remains almost the same. The stub has great impact on the second resonance frequency. Figure 9(b) depicts the effect of the height $h_{1}$ between the two arms and ground on $\left|S_{11}\right|$.

\subsection{Measured Results and Discussions}

To verify the simulations above, a prototype was built and measured. Figure 10 shows the geometry of the proposed dipole antenna prototype. For increasing the adhesion grounds located on the edge of the antenna which have

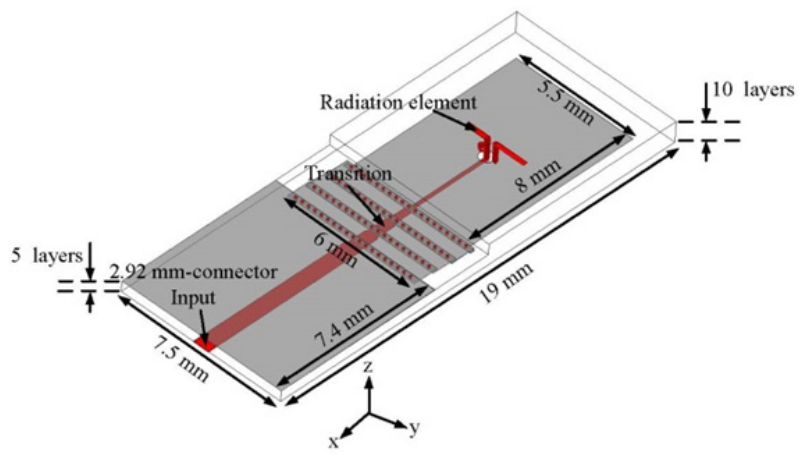

Fig. 10. Geometry of the dipole antenna prototype.
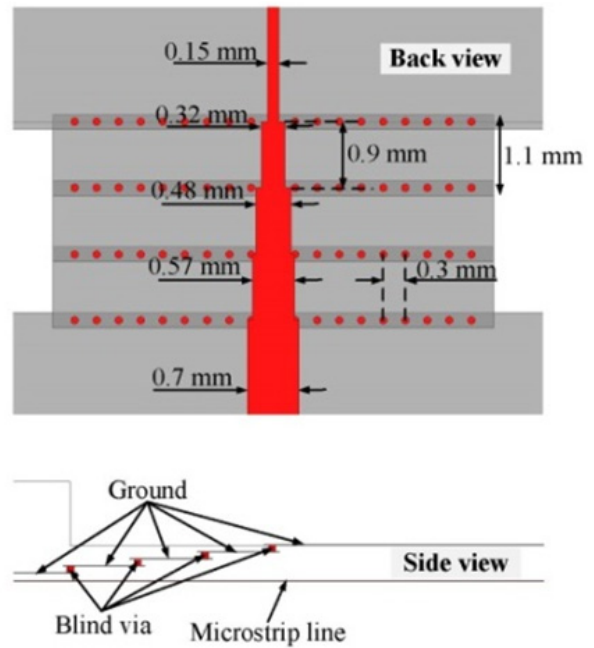

Fig. 11. Back and side views of the transition between microstrips with different thicknesses. 
little effect on the performance of the antenna were removed. The size of the antenna is $7.5 \times 19 \mathrm{~mm}^{2}$, and the thickness is $0.94 \mathrm{~mm}$.

A $2.92-\mathrm{mm}$ connector was used to measure the performance of the antenna, and in order to weld the 2.92mm connector easily, a transition between microstrips with different thicknesses, as shown in Fig .11, was designed to widen the width of the microstrip. The detailed design principle, process and simulated results had been illustrated in our paper [8], so they are not illustrated here for brevity.

The photographs of the dipole antenna prototype, measuring environment, simulated and measured $\left|S_{11}\right| \mathrm{s}$ and gains of the antenna are shown in Fig. 12. The meanings of the abbreviations are: sim: simulated, mea: measured, inc: including, exc: excluding, conn: 2.92-mm connector and $\tan \delta:$ the loss tangent of the substrate of the $2.92 \mathrm{~mm}$-connector.

As observed, the simulated $\left|S_{11}\right|$ of the antenna shown in Fig. 12 excluding the 2.92-mm connector is almost the same as that of the antenna shown in Fig. 2, the small difference between them is caused by the transition shown in Fig. 11 and the larger LTCC substrate around the dipole. The measured $\left|S_{11}\right|$ is better than the simulated one excluding the $2.92-\mathrm{mm}$ connector, which is mainly caused by the high loss of the transition from the microstrip to the 2.92-mm connector, because the reflected wave will be attenuated by twice.

For verifying this, the measured $\left|S_{11}\right|$ and $\left|S_{12}\right|$ of the back-to-back 2.92-mm connectors are shown in Fig. 13. The substrate, which is $30 \mathrm{~mm}$ in length, is RO4003C with dielectric permittivity $\varepsilon_{\mathrm{r}}=3.55$ and loss tangent $\tan \delta=0.0027$. The connector was welded by the same person under the same condition as it was welded in the prototype. The loss of the back-to-back 2.92-mm connectors is about $3 \mathrm{~dB}$. As a result, the loss of single connector is about $1.5 \mathrm{~dB}$ including $0.5-\mathrm{dB}$ loss of the connector itself, about $0.5-\mathrm{dB}$ simulated loss of the transition from the connector to the microstrip (the connector is set to be lossless) and $0.5-\mathrm{dB}$ welding error. This $1.5-\mathrm{dB}$ loss will improve the measured $\left|S_{11}\right|$. For easy understanding, the loss tangent of the substrate of the 2.92-mm connector is changed for introducing the 1.5-dB loss. As shown in Fig. 12, when $\tan \delta=0.1$, the simulated loss of the 2.92-mm connector is about $1.5 \mathrm{~dB}$. The simulated $\left|S_{11}\right|$ of the antenna including the $2.92-\mathrm{mm}$ connector when $\tan \delta=0.1$ is more like the measured one, which proves the $1.5-\mathrm{dB}$ loss of the 2.92 mm connector will improve the measured $\left|S_{11}\right|$ as shown in Fig. 12(b). The measured gain is plotted in Fig. 12(c). All the gains in this communication are realized gains. As observed, the measured gain excluding the connector agrees well with the simulated one when the frequency is higher than $31 \mathrm{GHz}$. The measured gain excluding the connector is lower than the simulated one when the frequency is lower than $31 \mathrm{GHz}$, which is because the measured $\left|S_{11}\right|$ below $31 \mathrm{GHz}$ is worse than the simulation as shown in Fig. 12(b).

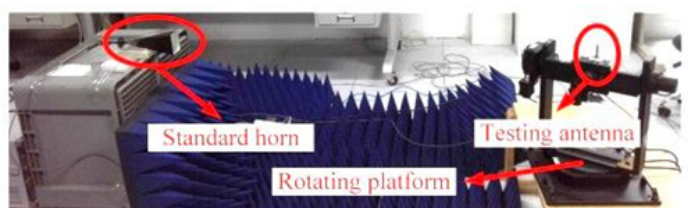

(a)

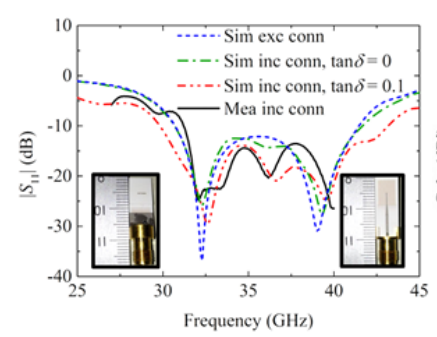

(b)

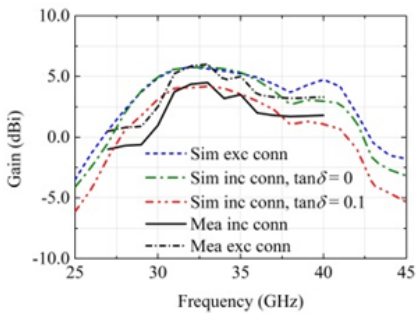

(c)
Fig. 12. (a) Measuring environment, (b) photographs of the antenna element prototype, simulated and measured $\left|S_{11}\right| \mathrm{s}$, and (c) simulated and measured gains.

The measured -10-dB $\left|S_{11}\right|$ bandwidth of the antenna is over $25.4 \%$ covering the frequency range of 31.2 to $40 \mathrm{GHz}$. Meanwhile, a maximum gain of $4.4 \mathrm{dBi}$ at $32 \mathrm{GHz}$ with a fluctuation of $1.7 \mathrm{~dB}$ over 31 to $39 \mathrm{GHz}$ frequency range is achieved. Simulated and measured radiation patterns of the antenna at $35 \mathrm{GHz}$ are indicated in Fig. 14. Good agreement is obtained between them.

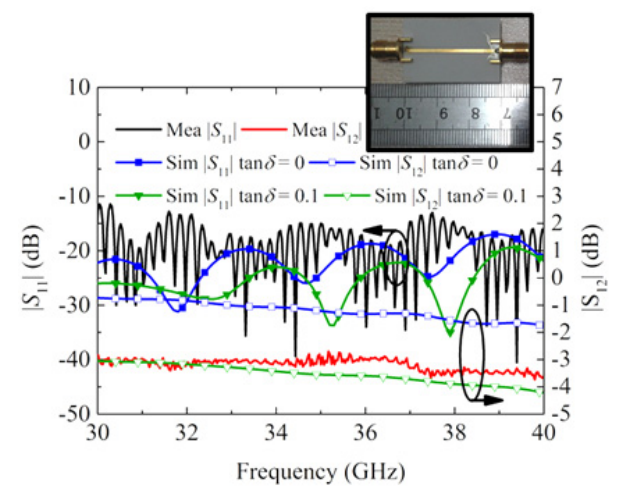

Fig. 13. Simulated and measured $\left|S_{11}\right|$ and $\left|S_{12}\right|$ of the backto-back 2.92-mm connectors.

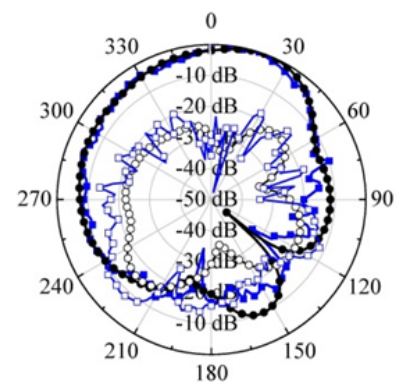

(a)

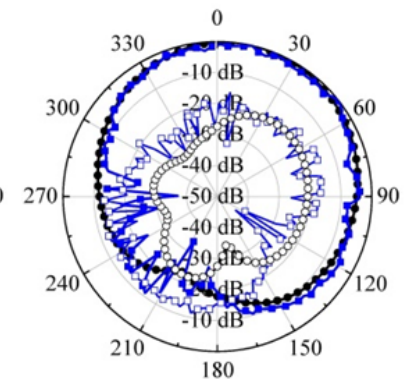

(b)
Fig. 14. Simulated and measured radiation patterns of the proposed dipole antenna at $35 \mathrm{GHz}$ : (a) XOZ-plane and (b) YOZ-plane. (blue, solid line, solid rectangle: mea co-pol; black, solid line, solid circle: sim co-pol; blue, solid line, hollow rectangle: mea cross-pol; black, solid line, hollow circle: sim cross-pol) 


\section{Antenna Array}

\subsection{Antenna Array Configuration}

A $2 \times 2$ array was also designed. Figure 15 shows the geometry of the proposed array. The design principle of the array is the same as that of the antenna element. The size of the array is $15 \times 25 \times 0.94 \mathrm{~mm}^{3}$. The inner-element spacing of the array is $4 \mathrm{~mm}\left(0.47 \lambda_{0}, \lambda_{0}\right.$ is the wavelength in free space at $35 \mathrm{GHz}$ ). A planar parallel microstrip feeding network was used to feed the $2 \times 2$ antenna elements with equal amplitude and phase. A 2.92-mm connector was also used to measure the performance of the proposed array.

\subsection{Measured Results and Discussions}

An array prototype was also built and measured. The photographs of the array prototype, measuring environment, simulated and measured $\left|S_{11}\right| \mathrm{s}$ and gains of the proposed array are shown in Fig. 16. As observed, the measured $\left|S_{11}\right|$ is also better than the simulated one excluding the 2.92-mm connector, which is also caused by the high loss of the transition from the microstrip to the 2.92-mm connector. The simulated $\left|S_{11}\right|$ of the array including the 2.92-mm connector when $\tan \delta=0.1$ is also more like the measured one, which proves the 1.5-dB loss of the 2.92mm connector will improve the measured $\left|S_{11}\right|$ again as shown in Fig. 16(b). The measured gain is plotted in Fig. 16(b). As observed, the measured gains excluding the connector agree well with the simulated ones when the frequency is higher than $31 \mathrm{GHz}$. The measured gain excluding the connector is higher than the simulated one when the frequency is lower than $31 \mathrm{GHz}$, because the beam width of the measured radiation pattern at $31 \mathrm{GHz}$ is narrower than the simulated ones as shown in Fig. 17(b).

The measured $-10-\mathrm{dB}\left|S_{11}\right|$ bandwidth is over $28.6 \%$ covering the frequency range of 30 to $40 \mathrm{GHz}$. Meanwhile, the array achieves a maximum gain of $10.37 \mathrm{dBi}$ at $31 \mathrm{GHz}$ with a fluctuation of $1.8 \mathrm{~dB}$ over $31-39 \mathrm{GHz}$ frequency range.

Simulated and measured radiation patterns of the array at 31,35 , and $39 \mathrm{GHz}$ are indicated in Fig. 17. The measured radiation patterns agree with the simulated ones at 35 and $39 \mathrm{GHz}$. The beam width of the measured radiation pattern at $31 \mathrm{GHz}$ is narrower than the simulated one.

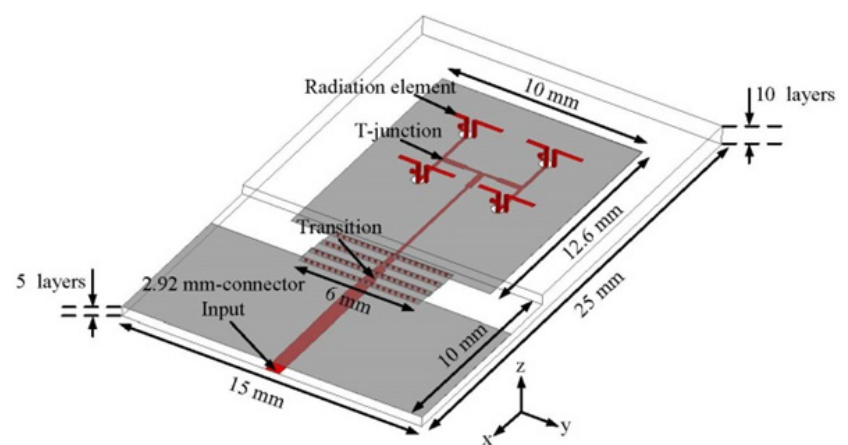

Fig. 15. Geometry of the array prototype.

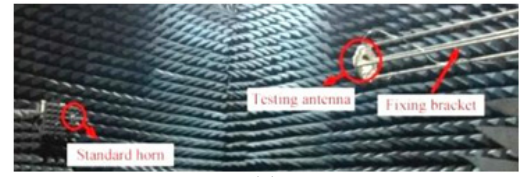

(a) (b)

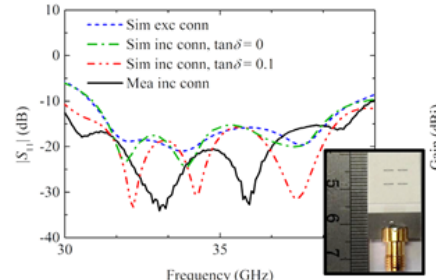

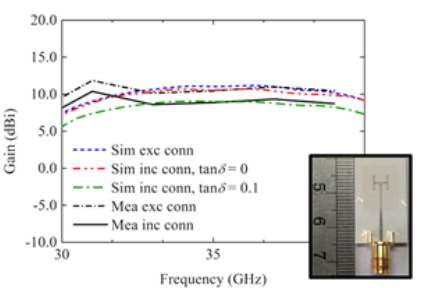

(c)
Fig. 16. (a) Measuring environment, (b) top view of the antenna array prototype, simulated and measured $\left|S_{11}\right| \mathrm{s}$, (c) back view of the antenna array prototype, simulated and measured gains.

The cross polarization increases with the increasing of frequency, because there are larger standing-wave currents on the circular aperture at higher frequencies. However, the cross polarization does not increase seriously because the circular aperture cannot radiate effectively.

Table 2 compares the performance of the proposed array and reported linearly polarized LTCC arrays for $\mathrm{mmW}$ applications. The proposed array provides the widest bandwidth.

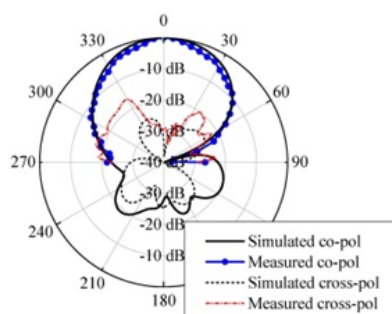

(a)

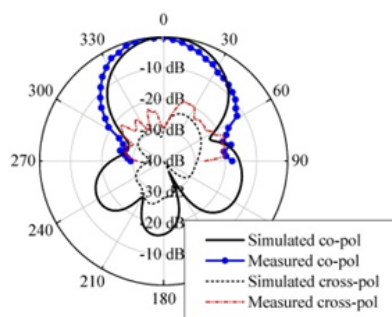

(c)

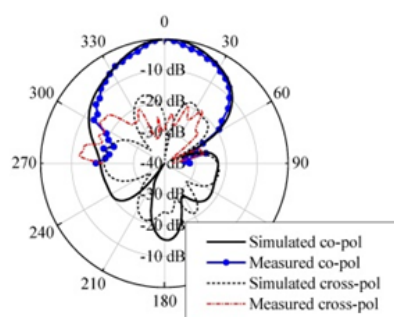

(e)

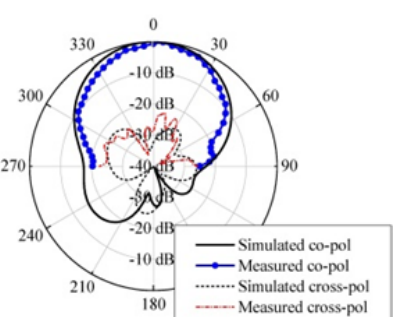

(b)

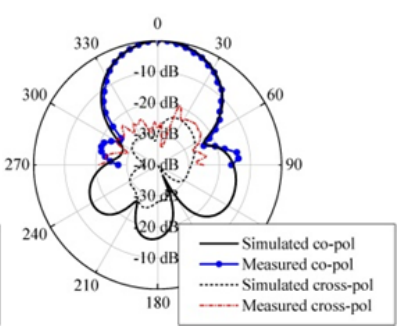

(d)

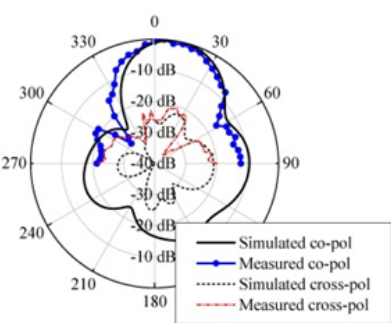

(f)
Fig. 17. Simulated and measured radiation patterns of the array: (a) XOZ-plane at $31 \mathrm{GHz}$, (b) YOZ-plane at $31 \mathrm{GHz}$, (c) XOZ-plane at $35 \mathrm{GHz}$, (d) YOZ-plane at $35 \mathrm{GHz}$, (e) XOZ-plane at $39 \mathrm{GHz}$ and (f) YOZ-plane at $39 \mathrm{GHz}$. 


\begin{tabular}{|c|c|c|c|c|c|}
\hline Ref. & Type & $\begin{array}{c}\text { Center } \\
\text { Freq. }\end{array}$ & Elements No. & Bandwidth & Gain \\
\hline$[1]$ & Patch & $60 \mathrm{GHz}$ & $4 \times 4$ & $7.8 \%$ & $17.2 \mathrm{dBi}$ \\
\hline$[5]$ & Patch & $60 \mathrm{GHz}$ & $2 \times 2$ & $23 \%$ & $9 \mathrm{dBi}$ \\
\hline$[21]$ & SIW cavity & $60 \mathrm{GHz}$ & $8 \times 8$ & $17.1 \%$ & $22.1 \mathrm{dBi}$ \\
\hline$[7]$ & Dipole & $60 \mathrm{GHz}$ & $4 \times 4$ & $15.7 \%$ & $15.6 \mathrm{dBi}$ \\
\hline$[8]$ & Dipole & $35 \mathrm{GHz}$ & $2 \times 2$ & $19.5 \%$ & $10 \mathrm{dBi}$ \\
\hline This work & Dipole & $35 \mathrm{GHz}$ & $2 \times 2$ & $28.6 \%$ & $10.37 \mathrm{dBi}$ \\
\hline
\end{tabular}

Tab. 2. Comparison to reported LTCC arrays.

\section{Conclusion}

Using LTCC technology, a 35-GHz vertical off-center-fed dipole antenna element with wideband fed by microstrip is proposed, fabricated and measured. A ringshaped stub was paralleled with a vertical off-center-fed dipole for wideband. Then, a $2 \times 2$ planar array fed with a microstrip feeding network was fabricated and measured. The proposed array exhibits wideband and high-gain characteristics.

\section{Acknowledgments}

This work was supported by the National Natural Science Foundation of China (No. 61401065), and China Scholarship Council (CSC).

\section{References}

[1] YEAP, S. B., CHEN, Z. N., QING, X. Gain-enhanced 60-GHz LTCC antenna array with open air cavities. IEEE Transactions on Antennas and Propagation, 2011, vol. 59, no. 9, p. 3470-3473. DOI: 10.1109/TAP.2011.2161549

[2] ZHANG, W., ZHANG, Y. P., SUN, M. et al. A 60-GHz circularlypolarized array antenna-in-package in LTCC technology. IEEE Transactions on Antennas and Propagation, 2013, vol. 61, no. 12, p. 6228-6232. DOI: 10.1109/TAP.2013.2279992

[3] WANG, L., GUO, Y. X., SHENG, W. X. Wideband high-gain 60$\mathrm{GHz}$ LTCC L-probe patch antenna array with a soft surface. IEEE Transactions on Antennas and Propagation, 2013, vol. 61, no. 4, p. 1802-1809. DOI: 10.1109/TAP.2012.2220331

[4] SUN, H., GUO, Y. X., WANG, Z. 60-GHz circularly polarized Uslot patch antenna array on LTCC. IEEE Transactions on Antennas and Propagation, 2013, vol. 61, no. 1, p. 430-435. DOI: 10.1109/TAP.2012.2214018

[5] CHIN, K. S., JIANG, W., CHE, W. et al. Wideband LTCC 60$\mathrm{GHz}$ antenna array with a dual-resonant slot and patch structure. IEEE Transactions on Antennas and Propagation, 2014, vol. 62 , no. 1, p. 174-182. DOI: 10.1109/TAP.2013.2287294

[6] YANG, W., WANG, H., CHE, W. Q., et al. High-gain and lowloss millimeter-wave LTCC antenna array using artificial magnetic conductor structure. IEEE Transactions on Antennas and Propagation, 2015, vol. 63, no. 1, p. 390-395. DOI: 10.1109/TAP.2014.2364591

[7] C. HUI, C., GUO, Y. X., WANG, Z. 60-GHz LTCC wideband vertical off-center dipole antenna and arrays. IEEE Transactions on Antennas and Propagation, 2013, vol. 61, no. 1, p. 153-161. DOI: 10.1109/TAP.2012.2220094
[8] DU, M., XU, J., DONG, Y.L., DING, X. LTCC SIW-vertical-feddipole array fed by microstrip network with tapered microstrip-toSIW transition for wideband millimeter-wave applications. IEEE Antennas and Wireless Propagation Letters, 2017, vol. 16, p. 1953-1956. DOI: 10.1109/LAWP.2017.2690325

[9] LIU, C., GUO, Y. X., BAO, X., XIAO, S. Q. 60-GHz LTCC integrated circularly polarized helical antenna array. IEEE Transactions on Antennas and Propagation, 2013, vol. 60, no. 3, p. 1329-1335. DOI: 10.1109/TAP.2011.2180351

[10] CAO, B., WANG, H., WANG, Z., et al. W-band LTCC helical antenna array with substrate integrated horn. In Asia-Pacific Microwave Conference (APMC). Sendai (Japan), 2014, p. 357-359. ISBN: 9784902339314

[11] WANG, X. Y., LI, J. L., ZHANG, Y. M., et al. A high-gain LTCC horn antenna with different feeding structures. In 2013 International Workshop on Microwave and Millimeter Wave Circuits and System Technology (MMWCST). Chengdu (China), 2013, p. 154-156. DOI: 10.1109/MMWCST.2013.6814593

[12] LEVINE, E., SHTRIKMAN, S., TREVES, D. Double-sided printed arrays with large bandwidth. IEE Proceedings $H$ Microwaves, Antennas and Propagation, 1988, vol. 135, no. 1, p. 54-59. DOI: 10.1049/ip-h-2.1988.0010

[13] EVTIOUSHKINE, G. A., KIM, J. W., HAN, K. S. Very wideband printed dipole antenna array. Electronics Letters, 1998, vol. 34 no. 24 , p. 2292-2293. DOI: 10.1049/el:19981615

[14] BAKHRAKH, L. D., LOS, V. F., SHAMANOV, A. N. Ultrawideband method of feeding a dipole antenna. In 4th International Conference on Antenna Theory and Techniques. Sevastopol (Ukraine), 2003, p. 535-538. DOI: 10.1109/ICATT.2003.1238795

[15] YIN, Y. Z., MA, J. P., ZHAO, Y. J., et al. Wideband printed dipole antenna for wireless LAN. In IEEE Antennas and Propagation Society International Symposium. Washington (USA), 2005, p. 568-571. DOI: 10.1109/APS.2005.1552074

[16] GAO, F., ZHANG, F., LU, L., et al. Low-profile dipole antenna with enhanced impedance and gain performance for wideband wireless applications. IEEE Antennas and Wireless Propagation Letters. 2013, vol. 12, p. 372-375. DOI: 10.1109/LAWP.2013.2252141

[17] TU, Z., ZHOU, D. F., ZHANG, G. Q., et al. A wideband cavitybacked elliptical printed dipole antenna with enhanced radiation patterns. IEEE Antennas and Wireless Propagation Letters. 2013, vol. 12, p. 1610-1613. DOI: 10.1109/LAWP.2013.2294058

[18] SHADROKH, S., YU, Y. Q., JOLANI, F., et al. Ultra-compact endloaded planar dipole antenna for ultra-wideband radar and communication applications. Electronics Letters, 2014, vol. 50, no. 21 , p. 1495-1496. DOI: 10.1049/el.2014.2008

[19] POZAR, D. M. Microwave Engineering, $4^{\text {nd }}$ ed. JohnWiley \& Sons, Inc., 2011. ISBN: 978-0-470-63155-3

[20] KRAUS, D. J., MARHEFKA, R. J. Antennas: For All Applications. $3^{\text {rd }}$ ed. New York (NY, USA): McGraw-Hill, 2002. ISBN-13: 978-0072321036

[21] XU, J., CHEN, Z. N., QING, X., HONG, W. Bandwidth enhancement for a $60 \mathrm{GHz}$ substrate integrated waveguide fed cavity array antenna on LTCC. IEEE Transactions on Antennas and Propagation, 2011, vol. 59, no. 3, p. 826-832. DOI: 10.1109/TAP.2010.2103018

\section{About the Authors ...}

Ming DU was born in China in 1989. He received the B.S. degree in Electrical Engineering from the University of Electronic Science and Technology of China (UESTC) 
Chengdu, China, in 2011, and is currently pursuing the Ph.D. degree in UESTC. His research interests include microwave circuits and antennas.

Jun XU was born in China in 1963. He received the B.S. degree in 1984, and M.S. degree in 1990 from the University of Electronic Science and Technology of China (UESTC), Chengdu, China. He engaged in basic research in the field of radio physics subject and teaching work. He was hired as an associate professor in 1997, then as a professor in 2000 in UESTC. His main research interests include microwave theory and technology, millimeter-wave hybrid integrated technology, millimeter-wave communication and radar radio frequency technology. He has published over 80 papers in refereed journals.

Xiao DING was born in Sichuan, China, in 1982. He received the Ph.D. degree in Radio Physics from the University of Electronic Science and Technology of China (UESTC), Chengdu, China, in 2013. He joined UESTC in 2014, where he is currently an associate professor. He has been a Visiting Scholar at the South Dakota School of Mines and Technology, SD, USA, a Visiting Fellow with the University of Houston, TX, USA. His research interests include wide-angle scanning phased array, LTCC millimeter wave array and reconfigurable antenna and its applications.

Jiangping CAO was born in Xinjiang, China, in 1981. She received the bachelor's degree in Mechanical Design, Manufacturing and Automation from the School of Mechanical Engineering of East China Jiaotong University, Nanchang, in 2004, and master's degree in Software Engineering from the University of Electronic Science and Technology of China in 2014. She is currently an engineer with the Na- tional Institute of Measurement and Testing Technology, China. Her research interests include the design and development of automation, electromechanical integration and intelligent equipment.

Jianhua DENG was born in Jiangxi, China, in 1977. He received the M.Sc. and Ph.D. degrees in Radio Physics from the University of Electronic Science and Technology of China (UESTC), Chengdu, China, in 2003 and 2007, respectively. From 2001 to 2006, he was a research assistant with the Millimeter-wave Laboratory, Southwest Institute of Electronic Technology of China, Chengdu, China. In 2009, he was an associate professor with the School of Physical Electronics, UESTC, and a research fellow with the Department of Electronic Engineering, City University of Hong Kong, Kowloon, Hong Kong. From 2010 to 2011, he was a post doctor with the Department of Electronic and Computer Engineering, Duke University, Durham, NC, USA. He is currently an associate professor with the National Institute of Measurement and Testing Technology, China. His current research interests are in the areas of optimization, modeling, and computer-aided design for microwave and millimeter-wave integrated circuits and antennas.

Yuliang DONG was born in Sichuan, China, in 1972. He received the B.S. degree in Electronic Engineering from the Northwestern Polytechnical University, Xi'an, China, in 1993, and Ph.D. degree from Beihang University, Beijing, in 2005. He is currently an associate professor with the National Institute of Measurement and Testing Technology, China. His research interests include microwave wave circuits, passive components, antennas and microwave CAD technology. 